\title{
Adjuvant Trastuzumab in HER2-Positive Breast Cancer
}

\author{
Dennis Slamon, M.D., Ph.D., Wolfgang Eiermann, M.D., Nicholas Robert, M.D., Tadeusz \\ Pienkowski, M.D., Miguel Martin, M.D., Michael Press, M.D., Ph.D., John Mackey, M.D., \\ John Glaspy, M.D., Arlene Chan, M.D., Marek Pawlicki, M.D., Tamas Pinter, M.D., Vicente \\ Valero, M.D., Mei-Ching Liu, M.D., Guido Sauter, M.D., Gunter von Minckwitz, M.D., Frances \\ Visco, J.D., Valerie Bee, M.Sc., Marc Buyse, Sc.D., Belguendouz Bendahmane, M.D., \\ Isabelle Tabah-Fisch, M.D., Mary-Ann Lindsay, Pharm.D., Alessandro Riva, M.D., John \\ Crown, M.D., and The Breast Cancer International Research Group* \\ The authors' affiliations are as follows: the Jonsson Comprehensive Cancer Center, University of \\ California-Los Angeles (D.S., J.G.) and the Norris Comprehensive Cancer Center, University of \\ Southern California (M.P.) - both in Los Angeles; Frauenklinik vom Roten Kreuz, Munich (W.E.), \\ the Department of Pathology, University Medical Center Hamburg-Eppendorf, Hamburg (G.S.), \\ and Zentrum für Frauenheilkunde, Frankfurt (G.M.) - all in Germany; U.S. Oncology Research \\ (N.R.) and University of Texas M.D. Anderson Cancer Center (V.V.) - both in Houston; Maria \\ Sklodowska-Curie Center, Warsaw, Poland (T. Pienkowski); Hospital Universitario San Carlos, \\ Madrid (M.M.); the Department of Oncology, University of Alberta, Edmonton, Canada (J.M.); \\ Mount Breast Group, Mount Hospital, Perth, Australia (A.C.); City Oncology Dispensary, St. \\ Petersburg, Russia (M.P.); Petz Oktato Korhaz Onkoradiologia, Budapest, Hungary (T. Pinter); \\ Sun Yat Sen Cancer Center, Taipei, Taiwan (M.-C.L.); National Breast Cancer Coalition, \\ Washington, DC (F.V.); Breast Cancer International Research Group (V.B., M.-A.L., A.R.) and \\ Sanofi-Aventis (B.B., I.T.-F.) - both in Paris; the International Drug Development Institute, \\ Louvain-la-Neuve, Belgium (M.B.); and All Ireland Cooperative Oncology Research Group, St. \\ Vincent's University Hospital, Dublin (J.C.)
}

\section{Abstract}

BACKGROUND_Trastuzumab improves survival in the adjuvant treatment of HER-positive breast cancer, although combined therapy with anthracycline-based regimens has been associated with cardiac toxicity. We wanted to evaluate the efficacy and safety of a new nonanthracycline regimen with trastuzumab.

METHODS-We randomly assigned 3222 women with HER2-positive early-stage breast cancer to receive doxorubicin and cyclophosphamide followed by docetaxel every 3 weeks (AC-T), the same regimen plus 52 weeks of trastuzumab (AC-T plus trastuzumab), or docetaxel and carboplatin plus 52 weeks of trastuzumab $(\mathrm{TCH})$. The primary study end point was disease-free survival. Secondary end points were overall survival and safety.

RESULTS-At a median follow-up of 65 months, 656 events triggered this protocol-specified analysis. The estimated disease-free survival rates at 5 years were $75 \%$ among patients receiving AC-T, 84\% among those receiving AC-T plus trastuzumab, and 81\% among those receiving $\mathrm{TCH}$. Estimated rates of overall survival were $87 \%, 92 \%$, and $91 \%$, respectively. No significant differences in efficacy (disease-free or overall survival) were found between the two trastuzumab

Copyright @ 2011 Massachusetts Medical Society

Address reprint requests to Dr. Slamon at the University of California-Los Angeles, 10945 Le Conte Ave., Suite 3360, Los Angeles, CA 90095-1678, or at dslamon@mednet.ucla.edu..

*Additional investigators in the Breast Cancer International Research Group (BCIRG) 006 study are listed in the Supplementary Appendix, available at NEJM.org.

Disclosure forms provided by the authors are available with the full text of this article at NEJM.org. 
regimens, whereas both were superior to AC-T. The rates of congestive heart failure and cardiac dysfunction were significantly higher in the group receiving AC-T plus trastuzumab than in the TCH group $(\mathrm{P}<0.001)$. Eight cases of acute leukemia were reported: seven in the groups receiving the anthracycline-based regimens and one in the TCH group subsequent to receiving an anthracycline outside the study.

CONCLUSIONS-The addition of 1 year of adjuvant trastuzumab significantly improved disease-free and overall survival among women with HER2-positive breast cancer. The riskbenefit ratio favored the nonanthracycline $\mathrm{TCH}$ regimen over AC-T plus trastuzumab, given its similar efficacy, fewer acute toxic effects, and lower risks of cardiotoxicity and leukemia. (Funded by Sanofi-Aventis and Genentech; BCIRG-006 ClinicalTrials.gov number, NCT00021255.)

The her2 gene encodes a tyrosine kinase receptor that mediates critical signaling functions in normal and malignant breast epithelial cells. ${ }^{1}$ An acquired alteration consisting of amplification and overexpression of the gene product occurs in approximately 20 to $25 \%$ of human breast cancers. ${ }^{2,3}$ HER2 overexpression is associated with an aggressive clinical pheno-type that includes high-grade tumors, increased growth rates, early systemic metastasis, and decreased rates of disease-free and overall survival., ${ }^{2,3}$ Preclinical data indicate that this adverse clinical picture results from fundamental changes in the biologic features of breast-cancer cells containing the alteration, including increased proliferation, suppression of apoptosis, increased motility, greater invasive and metastatic potential, accelerated angiogenesis, and steroid hormone independence. ${ }^{4-11}$

In previous studies, many of these HER2-mediated adverse characteristics were reversed by the use of monoclonal antibodies directed against the tyrosine kinase receptor, ${ }^{10,12-15}$ and these data led to phase 1 testing of a murine anti-HER2 monoclonal antibody, 4D5. ${ }^{16}$ Preliminary efficacy and safety data prompted the development of a humanized monoclonal antibody to produce trastuzumab. ${ }^{17}$ Alone and in combination with chemotherapy, trastuzumab has been shown to have an acceptable safety record and to be active in advanced HER2-positive disease. ${ }^{18-20}$ Subsequently, in a large, randomized study, the addition of trastuzumab to chemotherapy yielded significant improvements in rates of objective response, response duration, and time to disease progression $(56 \%, 58 \%$, and $65 \%$ improvement, respectively), as well as a $30 \%$ improvement in the rate of overall survival among patients with first-line metastatic disease. ${ }^{21}$ A significant side effect was an increase by a factor of 4 in the rate of cardiac dysfunction, including congestive heart failure, especially when trastuzumab was used in combination with anthracycline-based regimens. ${ }^{21}$

These data led to the initial regulatory approval of trastuzumab for metastatic HER2-positive breast cancer and resulted in its evaluation in early-stage disease. Five randomized trials (four large and one small) were then launched to evaluate the efficacy and safety of adjuvant therapy with trastuzumab, and findings in three of these trials have been reported. ${ }^{22-24}$ One report contained results from similar treatment groups in two studies. ${ }^{23}$ All three large studies used trastuzumab in combination either exclusively or predominantly with anthracycline-based regimens, ${ }^{22,23}$ and all showed a significant benefit of trastuzumab, with a reduction in the rate of recurrence of approximately 50\% and improvement in the rate of survival of approximately $30 \% .22,23$ Again, an increase by a factor of 4 to 5 in the rate of congestive heart failure was noted when adjuvant trastuzumab was used with anthracyclines, and an even larger proportion of patients had subclinical loss of left ventricular function. ${ }^{22,23,25,26}$

In the fourth large study, the Breast Cancer International Research Group 006 (BCIRG-006) trial, we also evaluated adjuvant therapy with trastuzumab but included a second trastuzumab-containing regimen, which did not have an anthracycline. This new regimen, consisting of docetaxel, carboplatin, and trastuzumab (TCH), is based on preclinical 
synergies observed between trastuzumab and platinum salts or docetaxel that were not seen with anthracyclines or paclitaxel. ${ }^{14,27,28}$ It was also anticipated that TCH might circumvent the cardiac toxicity seen with anthracycline-based regimens. ${ }^{21}$ Phase 2 and 3 trials of TCH in patients with advanced disease showed that this regimen resulted in the longest period of progression-free survival reported to date, with rare cardiac dysfunction. ${ }^{18-20} \mathrm{~A}$ trial comparing TCH with docetaxel plus trastuzumab in metastatic disease showed no significant difference in progression-free survival between these two regimens. ${ }^{29}$ However, the dose of docetaxel was $33 \%$ higher in the group receiving docetaxel plus trastuzumab than in the group receiving TCH (100 mg per square meter of body-surface area vs. $75 \mathrm{mg}$ per square meter). The only trial that directly evaluated the added benefit of carboplatin in a doseequivalent, taxane-containing regimen showed that TCH had a significant advantage with respect to progression-free survival over paclitaxel plus trastuzumab. ${ }^{19}$ For these reasons, we decided to evaluate TCH in addition to an anthracycline-based regimen in adjuvant treatment of HER2-positive breast cancers. We report efficacy and safety results from this study in an intention-to-treat analysis of data from all 3222 patients.

\section{METHODS}

\section{PATIENTS AND STUDY DESIGN}

From April 2001 through March 2004, we randomly assigned 3222 women with HER2positive, invasive, high-risk, node-negative or node-positive adenocarcinoma (stage T1, T2, or T3) from 41 countries to receive a standard adjuvant anthracycline-taxane chemotherapy regimen, the same regimen plus trastuzumab, or a new non-anthracycline, trastuzumabbased regimen (Fig. 1 in the Supplementary Appendix, available with the full text of this article at NEJM.org). Specific demographic and clinical characteristics of patients were similar in the three study groups (Table 1). The HER2-positive status of all tumors was confirmed with the use of fluorescence in situ hybridization (PathVision, Abbott) before randomization.

In the first group, women received standard therapy with doxorubicin (60 mg per square meter) and cyclophosphamide (600 mg per square meter) every 3 weeks for four cycles, followed by docetaxel (100 mg per square meter) every 3 weeks for four doses (AC-T). In the second group, patients received AC-T plus trastuzumab, beginning with the first dose of docetaxel and continuing for 1 year (AC-T plus trastuzumab). In the third group, patients received docetaxel ( $75 \mathrm{mg}$ per square meter) plus carboplatin (area under the curve, $6 \mathrm{mg}$ per milliliter per minute), given every 3 weeks for six cycles concurrently with trastuzumab, followed by trastuzumab for an additional 34 weeks (TCH). In the two trastuzumabcontaining regimens, trastuzumab was initially administered at a dose of $4 \mathrm{mg}$ per kilogram of body weight, followed by $2 \mathrm{mg}$ per kilogram per week during chemotherapy and then 6 $\mathrm{mg}$ per kilogram every 3 weeks to complete 1 year of trastuzumab treatment. Hematopoietic growth factors were used at the discretion of treating physicians.

The institutional review board at each institution approved the study. All patients provided written informed consent.

\section{PRIMARY AND SECONDARY END POINTS}

The study's primary end point was disease-free survival, which was defined as the time from randomization to breast-cancer recurrence, a second primary cancer (excluding contralateral ductal carcinoma in situ), or death from any cause, whichever came first. Secondary end points included overall survival, global safety, and cardiac safety. 


\section{CARDIAC SAFETY}

In addition to monitoring general safety, we instituted an intense cardiac-monitoring schedule. We measured the left ventricular ejection fraction (LVEF) in each patient seven times throughout the study to detect congestive heart failure or sustained asymptomatic subclinical loss of mean LVEF, which was defined as a relative reduction from baseline of more than $10 \%$ at the last follow-up evaluation (Section 2 in the Supplementary Appendix). An independent cardiac review panel whose members were unaware of study-group assignments reviewed all cases of suspected cardiac events. In addition, we calculated and reported the mean LVEF in each study group, measured at protocol-defined times, along with $95 \%$ confidence intervals.

\section{TOP2A COAMPLIFICATION}

We also evaluated the effect on efficacy of a second alteration within the HER2 amplicon that imparts incremental anthracycline sensitivity to a subset of HER2-positive cancers. The prespecified analysis of the gene encoding topoisomerase II alpha (TOP2A) showed that coamplification of this gene occurs in 35\% of HER2-positive cancers. These data, together with the methods used, have been reported previously. ${ }^{30}$ When present, TOP2A coamplification is associated with the incremental benefit previously described for anthracycline as compared with nonanthracycline regimens in HER2-positive breast cancers. $^{31,32}$

\section{STUDY OVERSIGHT}

The study was conducted in accordance with the protocol, which is available at NEJM.org. The breast steering committee of the BCIRG study group, which was composed entirely of academic investigators, designed the study and collected and analyzed all the data. The trial advisory committee consisted of one member from each sponsor and six members of the BCIRG breast steering committee. The resulting report was written by the first author and reviewed by the steering committee, who made the decision to submit the manuscript for publication. All authors vouch for the accuracy and completeness of the data. The sponsor and major funder of the study was Sanofi-Aventis, with additional support provided by Genentech. Trastuzumab was provided by Genentech free of charge for study patients in the United States and was purchased by Sanofi-Aventis for all study patients in other countries. Docetaxel was provided by Sanofi-Aventis for all study patients.

\section{STATISTICAL ANALYSIS}

We used log-rank tests to compare disease-free survival and overall survival in the three study groups and plotted Kaplan-Meier product-limit estimators. We planned to conduct three protocol-specified interim analyses and a final analysis after observing 300, 450, 650, and 900 events, respectively, according to an O'Brien-Fleming group sequential boundary. The significance levels (alpha) for these analyses were equal to approximately 0.0002 , $0.0030,0.0111$, and 0.0461 , respectively. At each analysis, a step-down testing procedure was used. We compared the AC-T group with each trastuzumab-containing regimen (AC-T plus trastuzumab and $\mathrm{TCH}$ ) at a significance level equal to one half alpha to account for multiple testing. If both comparisons were significant, we compared the two trastuzumab regimens at a significance equal to alpha.

The first interim analysis was carried out after the observation of 322 disease-free survival events in September 2005. At that time, the efficacy boundaries were crossed and showed a significant difference in disease-free survival events in favor of both AC-T plus trastuzumab and $\mathrm{TCH}$, as compared with AC-T, with no significant difference between AC-T plus trastuzumab and $\mathrm{TCH}$. Here, we present the results of the third protocol-specified analysis. 
For this protocol-specified analysis (656 events), the study had a power of $80 \%$ to detect a hazard ratio of 0.75 or less for the comparison of either of the two trastuzumab-containing regimens with AC-T and a power of $75 \%$ to detect a hazard ratio of 0.64 or less for the comparison of AC-T plus trastuzumab with TCH. All reported P values are two-sided. All analyses were conducted by the International Institute for Drug Development and were performed with the use of SAS statistical software, version 9.0 (SAS Institute). The results of all analyses were presented to an independent data and safety monitoring committee.

\section{RESULTS}

\section{PRIMARY AND SECONDARY END POINTS}

At the time of this analysis, 656 disease-free survival events were observed (257 in the group receiving AC-T, 185 in the group receiving AC-T plus trastuzumab, and 214 in the group receiving TCH). At this point, 348 patients had died during a median follow-up of 65 months. For both primary and secondary efficacy end points, a significant benefit with respect to disease-free and overall survival was seen in both groups treated with trastuzumab-containing regimens, as compared with the group that received AC-T (standard therapy), which had a 5-year rate of disease-free survival of 75\% (Fig. 1A) and a rate of overall survival of $87 \%$ (Fig. 2 in the Supplementary Appendix). For patients receiving AC$\mathrm{T}$ plus trastuzumab, the 5-year rate of disease-free survival was $84 \%$ (hazard ratio for the comparison with AC-T, 0.64; $\mathrm{P}<0.001$ ), and the rate of overall survival was $92 \%$ (hazard ratio, $0.63 ; \mathrm{P}<0.001)$. For patients receiving $\mathrm{TCH}$, the 5 -year rate of disease-free survival was $81 \%$ (hazard ratio, $0.75 ; \mathrm{P}=0.04$ ), and the rate of overall survival was $91 \%$ (hazard ratio, 0.77; $\mathrm{P}=0.04)$. In contrast, no significant difference in the rate of disease-free or overall survival was seen between the two trastuzumab-containing regimens. However, the study was not powered to detect equivalence between these two regimens.

Analyses of absolute between-group differences, stratified according to nodal status and tumor size, were also performed. These analyses showed that among node-negative patients, the estimated rates of disease-free survival at 5 years were significantly improved by trastuzumab, with a rate of $93 \%$ in the group receiving AC-T plus trastuzumab and $90 \%$ in the group receiving $\mathrm{TCH}$, as compared with $85 \%$ in the group receiving $\mathrm{AC}-\mathrm{T}$ (hazard ratio for AC-T plus trastuzumab, 0.47; $\mathrm{P}=0.003$; hazard ratio for TCH, 0.64; $\mathrm{P}=0.06$ ) (Table $1 \mathrm{~A}$ in the Supplementary Appendix).

Among node-positive patients, trastuzumab also significantly improved 5-year rates of disease-free survival, which were $80 \%$ in the group receiving AC-T plus trastuzumab and $78 \%$ in the group receiving $\mathrm{TCH}$, as compared with $71 \%$ in the group receiving AC-T (hazard ratio for AC-T plus trastuzumab, 0.68; $\mathrm{P}<0.001$; hazard ratio for $\mathrm{TCH}, 0.78 ; \mathrm{P}=$ $0.01)$ (Table $1 \mathrm{~A}$ in the Supplementary Appendix). In addition, a benefit of trastuzumab was seen in node-positive patients at highest risk for recurrence (i.e., those with $\geq 4$ positive nodes), for whom the 5-year rate of disease-free survival was $73 \%$ in the group receiving AC-T plus trastuzumab and $72 \%$ in the group receiving $\mathrm{TCH}$, as compared with $61 \%$ in the group receiving $\mathrm{AC}-\mathrm{T}$ (hazard ratio, $0.66 ; \mathrm{P}=0.002$ for both comparisons) (Table $1 \mathrm{~A}$ in the Supplementary Appendix).

Analysis of the efficacy of trastuzumab on the basis of the size of the primary tumor showed similarly significant benefits for patients with small or large primary tumors, with no significant between-group differences among patients with tumors of intermediate size (Table 1B in the Supplementary Appendix). Among patients with HER2-positive tumors measuring $1 \mathrm{~cm}$ or less in the greatest diameter, the estimated 5-year rates of disease-free survival were $86 \%$ in the group receiving AC-T plus trastuzumab and $86 \%$ in the group receiving $\mathrm{TCH}$, as compared with $72 \%$ in the group receiving AC-T (hazard ratio for AC-T 
plus trastuzumab, 0.36; $\mathrm{P}=0.03$; hazard ratio for TCH, 0.45; $\mathrm{P}=0.09$ ) (Table 1B in the Supplementary Appendix). For tumors measuring $1 \mathrm{~cm}$ or more but no more than $2 \mathrm{~cm}$, the analysis showed no significant difference in 5-year rates of disease-free survival, with rates of $87 \%$ in the group receiving AC-T plus trastuzumab and $86 \%$ in the group receiving $\mathrm{TCH}$, as compared with $86 \%$ in the group receiving AC-T (hazard ratio for AC-T plus trastuzumab, 0.88; $\mathrm{P}=0.59$; hazard ratio for $\mathrm{TCH}, 1.11 ; \mathrm{P}=0.64$ ) (Table 1B in the Supplementary Appendix). For tumors measuring $2 \mathrm{~cm}$ or more at diagnosis, the 5-year rates of disease-free survival were $82 \%$ in the group receiving AC-T plus trastuzumab and $79 \%$ in the group receiving $\mathrm{TCH}$, as compared with $71 \%$ in the group receiving $\mathrm{AC}-\mathrm{T}$ (hazard ratio for AC-T plus trastuzumab, 0.62; $\mathrm{P}<0.001$; hazard ratio for TCH, 0.70; $\mathrm{P}<0.001$ ) (Table 1B in the Supplementary Appendix). Forest-plot analyses also showed improved efficacy for both trastuzumab-containing regimens, as compared with standard therapy, with respect to rates of disease-free and overall survival in subgroups defined according to nodal status (negative vs. positive), hormone-receptor results (negative vs. positive), and tumor size ( $\leq 2$ cm vs. $>2 \mathrm{~cm}$ ) (Fig. 3A through 3D in the Supplementary Appendix).

Overall, among the 3222 patients who underwent randomization, there were only 29 more primary events in the TCH group (214 events) than in the group receiving AC-T plus trastuzumab (185 events). A comparison of the therapeutic indexes of the two trastuzumabcontaining regimens and the standard-therapy regimen was performed on the basis of the numbers of distant breast-cancer recurrences, cases of congestive heart failure, and cases of acute leukemia occurring in each study group (Table 2). In this comparison, the difference between the trastuzumab-containing regimens was even smaller than the difference in the number of primary events.

\section{TOP2A Coamplification}

Among patients with HER2-positive breast cancers without TOP2A coamplification, the diseasefree survival benefit with trastuzumab-containing regimens was even greater than that seen in the overall analysis of all patients (191 patients in the group receiving AC-T, 119 in the group receiving AC-T plus trastuzumab, and 130 in the group receiving TCH) (Fig. 1A and 1B). However, in the 35\% of HER2-positive cancers in which TOP2A was coamplified, the rates of disease-free survival for the trastuzumab-containing regimens were virtually indistinguishable from the rate with standard therapy (55 patients in the group receiving AC-T, 52 in the group receiving AC-T plus trastuzumab, and 72 in the group receiving $\mathrm{TCH}$ ) (Fig. 1C). This subpopulation of patients appears to derive no incremental benefit from trastuzumab, even though the therapeutic index favors TCH over the anthracycline-based regimen because of better short- and long-term toxicity profiles (Tables 2,3 , and 4).

\section{Adverse Effects}

General Safety-We monitored side effects of the adjuvant chemotherapy regimens as well as specific toxic effects that were linked to individual agents. All toxic effects were graded according to the National Cancer Institute Common Toxicity Criteria (version 2.0). A significant difference favoring the group receiving $\mathrm{TCH}$, as compared with the group receiving AC-T plus trastuzumab, was noted for arthralgias, myalgias, the hand-foot syndrome, stomatitis, and vomiting (Table 3). Significant differences in sensory and motor neuropathies, nail changes, and myalgias also favored the TCH group. The incidences of neutropenia and leukopenia were significantly lower in the TCH group than in the group receiving AC-T plus trastuzumab, whereas the incidences of anemia and thrombocytopenia were significantly lower in the group receiving AC-T plus trastuzumab than in the TCH group. Finally, acute leukemias developed in seven patients who were receiving anthracycline-based regimens and in one patient who was receiving TCH. However, in this 
patient, leukemia was diagnosed after the patient had received an anthracycline for the treatment of a B-cell lymphoma that occurred after her breast cancer. None of these patients had a breast-cancer recurrence, and at the time of this report, five of the eight patients with leukemia had died.

Cardiac Safety-At study entry, cardiac risk factors were well balanced among the three study groups (Table 4). At the time of this analysis, the incidence of congestive heart failure in the two trastuzumab-containing regimens was higher in the group receiving AC-T plus trastuzumab $(2.0 \%)$ than in the AC-T group $(0.7 \%)$ or the TCH group $(0.4 \%)$; the incidence with AC-T plus trastuzumab as compared with $\mathrm{TCH}$ was increased by a factor of 5 . The difference in rates of congestive heart failure between the two trastuzumab-containing regimens significantly favored TCH over AC-T plus trastuzumab $(\mathrm{P}<0.001)$. In addition, a significant difference in sustained, subclinical loss of mean LVEF (defined as $>10 \%$ relative loss) was observed in the group receiving AC-T plus trastuzumab, as compared with the TCH group (18.6\% vs. $9.4 \%, \mathrm{P}<0.001)$, with a rate of $11.2 \%$ in the AC-T group. Although the cardiac dysfunction associated with trastuzumab after the use of adjuvant anthracyclines has been reported to be relatively transient, ${ }^{22-26}$ in this study, the subclinical toxic effect persisted for several years (Fig. 2). Of 194 of the 1042 patients (19\%) who had a relative reduction in LVEF of more than $10 \%$ in the group receiving AC-T plus trastuzumab, the decrease persisted for at least 4 years after randomization in $33 \%$ of the patients. Of note, 23 of 1073 patients $(2.1 \%)$ who were assigned to receive AC-T plus trastuzumab never received trastuzumab because the LVEF declined to an unacceptable level after the initial anthracycline treatment. Consequently, these HER2-positive women received no targeted therapy after receiving anthracycline therapy.

\section{Discussion}

The rationale supporting the adjuvant use of trastuzumab is derived from an improved understanding of the molecular diversity of human breast cancer $2,3,21,33$ and recognition that this disease should not be treated with the one-size-fits-all approach that has characterized most approaches to adjuvant chemotherapy for the past four decades. The significant efficacy shown by trastuzumab in treating first-line metastatic HER2-positive breast cancer $^{21}$ prompted its evaluation in early-stage disease. This study is one of four large, randomized adjuvant trials undertaken as part of this evaluation. Like the previously reported studies, ${ }^{22-24}$ our study showed improvements in efficacy with the adjuvant use of trastuzumab. The major safety issue that we observed was increased cardiac dysfunction when trastuzumab was used in combination with anthracycline-based chemotherapy, a finding that is also concordant with the results of previous studies. $22,23,25,26$

Despite these similarities, there are three distinct differences between our study and the other trials of adjuvant trastuzumab. First, we performed the intention-to-treat analyses of efficacy and safety as originally and prospectively defined in the study protocol; no statistical modifications or study-group exclusions were permitted for combined or early analyses of data. Second, after the initial efficacy results for adjuvant trastuzumab were reported in April 2005, only 23 patients (2.1\%) in the control group (i.e., those receiving AC-T) crossed over to receive trastuzumab before reaching prespecified study end points. This permitted unbiased long-term comparisons of both efficacy and safety among the three study groups. Third, and perhaps most important, our study was the only one to include a nonanthracycline chemotherapy regimen.

Almost all current adjuvant therapies incorporate the use of anthracyclines on the basis of the meta-analysis by the Early Breast Cancer Trialists' Group, which showed an improved efficacy of anthracycline-containing chemotherapy regimens over regimens without an 
anthracycline. ${ }^{34}$ However, analyses of HER2 status among approximately 5200 patients who participated in eight large, randomized adjuvant trials comparing anthracycline with nonanthracycline chemotherapy regimens showed that only women with HER2-positive breast cancer derived an incremental benefit from anthracycline use. ${ }^{35-39}$ No incremental benefit was apparent for the 75 to $80 \%$ of breast cancers that were HER2-negative. A metaanalysis of these and additional data has been reported. ${ }^{40}$ Detailed data derived from an analysis of the TOP $2 A$ gene in our study showed that TOP $2 A$ coamplification occurred in $35 \%$ of HER2-positive patients and was directly associated with the incremental benefit that anthracyclines provide over nonanthracycline regimens in HER2-positive disease. ${ }^{30}$ The use of a non-anthracycline $(\mathrm{TCH})$ regimen in our study allowed us to ask whether preclinical synergies observed between platinum-based chemotherapy and trastuzumab, as well as between docetaxel and trastuzumab, ${ }^{15,27}$ might compensate for the incremental efficacy advantage of anthracyclines in HER2-positive breast cancers. Although our study was not powered to detect equivalence between the two trastuzumab-containing groups, outcome data showed that TCH had similar efficacy (with respect to disease-free and overall survival) despite the omission of anthracyclines and the use of two rather than three cytotoxic drugs. In our study, this phenomenon was shown even in patients who are thought to be at highest risk for recurrence (i.e., those with $\geq 4$ positive lymph nodes or a primary tumor measuring $>2 \mathrm{~cm}$ at diagnosis). Moreover, although the overall numerical advantage (29 disease-free survival events) in favor of AC-T plus trastuzumab over TCH was not significant, this difference in efficacy came at the cost of a highly significant increase in congestive heart failure ( 21 cases vs. 4 cases). These differences in rates of congestive heart failure were not taken into account in any of the protocol-specified efficacy comparisons. In addition, our findings show that the use of AC-T plus trastuzumab resulted in a significantly increased risk of sustained subclinical loss in LVEF. Finally, the use of TCH had the pragmatic advantage of allowing patients to complete all adjuvant chemotherapy in 12 rather than 16 weeks, as well as allowing them to complete 12 months of trastuzumab therapy 12 weeks sooner and requiring fewer infusion visits.

The emerging further understanding of long-term and life-altering toxic effects associated with adjuvant anthracyclines may provide the most compelling support for nonanthracycline regimens. The well-known, long-term side effects of anthracyclines include significantly increased risks of congestive heart failure, ${ }^{41,42}$ myelodysplasia, and acute leukemia. ${ }^{43}$ However, recent analyses of the National Cancer Institute Surveillance, Epidemiology, and End Results (SEER) database indicate that we may be underestimating the full effect of anthracycline use on long-term cardiac and hematologic health in patients treated for breast cancer with these agents, ${ }^{41-43}$ possibly because the majority of adjuvant studies have been designed primarily to evaluate differences in efficacy and have used follow-up periods of 7 to 10 years for both efficacy and safety analyses. Attempts to obtain longer-term safety data, including cardiac outcomes, are systematically biased by loss of later follow-up.

In addition, the SEER database is derived from information on women 65 years of age or older. The mean age of women in the HER2-positive adjuvant studies was 51 to 52 years, whereas the mean age of the overall breast-cancer population is approximately 62 years. Given that data from our study and other trials $22,23,25,26$ show that trastuzumab augments the incidence of anthracycline-associated congestive heart failure and subclinical loss of LVEF, the full effect of any nonsymptomatic damage induced by the combination of trastuzumab with anthracycline-based regimens may not be apparent until much later as these younger women have additional, age-related cardiac insults. Our findings on long-term, subclinical LVEF losses, as well as the published results from the SEER database analyses, ${ }^{41-43}$ involving some 42,000 women, support this concern. 
Furthermore, although myeloid growth factors permit delivery of full-dose, acceleratedschedule adjuvant anthracycline-cyclophosphamide treatments, registry data suggest a doubling of the incidences of acute leukemia and myelodysplasia associated with these regimens, though the absolute risk remains low. ${ }^{43}$ Of note, all cases of acute leukemia in our study occurred in patients who had previously been exposed to an anthracycline. Some observers have argued that these major toxic effects are rare and are offset by significant efficacy gains obtained with anthracyclines. However, our data do not fully support this argument. We did not find any significant incremental therapeutic benefits of combined trastuzumab-anthracycline treatment as compared with $\mathrm{TCH}$, yet we did see significant increases in both acute and chronic toxic effects with the trastuzumab-anthracycline regimen. Consequently, we believe that $\mathrm{TCH}$ offers an effective alternative to the anthracycline-based regimens and their associated risks.

An improved understanding of the molecular basis of malignant disease is allowing the development of rational treatment strategies that are more effective and less toxic than traditional empiric regimens. The identification and characterization of the HER2 alteration in a subset of human breast cancers and the subsequent development of trastuzumab represent the practical realization of this translational ideal. Our findings show that we can further exploit this new translational knowledge to optimize efficacy while simultaneously minimizing acute and chronic toxic effects in the adjuvant treatment of HER2-positive breast cancer.

\section{Supplementary Material}

Refer to Web version on PubMed Central for supplementary material.

\section{Acknowledgments}

Supported by Sanofi-Aventis and Genentech; a Department of Defense Breast Cancer Innovator Award and funding from the Revlon/UCLA Women's Cancer Program and the Peter and Denise Wittich Breast Cancer Program (to Dr. Slamon); and grants (to Dr. Press) from the U.S. Army Medical Research and Development Command (DAMD-03-1-0626), the National Cancer Institute (CA 48780), and the California Breast Cancer Research Program (12IB-0155 and 14NB-0179) for studies of TOP2A.

Dr. Slamon reports receiving honoraria and reimbursement for travel expenses from Genentech and Sanofi-Aventis; Dr. Eiermann, receiving honoraria, lecture fees, and reimbursement for travel expenses from Roche and SanofiAventis; Dr. Robert, receiving reimbursement for travel expenses from Fairfax Northern Virginia Hematology Oncology PC and that his institution has received grant support, honoraria, and payments for the development of educational presentations from Fairfax Northern Virginia Hematology Oncology PC on his behalf; Dr. Pienkowski, serving as a board member for Sanofi-Aventis and Roche, receiving honoraria from Roche and GlaxoSmithKline, and that he and his institution have received lecture fees and reimbursement for travel expenses from Roche and Sanofi-Aventis; Dr. Press, receiving consulting fees, honoraria, lecture fees, and reimbursement for travel expenses from Genentech; Dr. Mackey, receiving honoraria from Roche; Dr. Glaspy, receiving lecture fees from Genentech, Amgen, and Eli Lilly; Dr. Chan, serving on an advisory board for and receiving consulting fees, grant support, and honoraria from Roche and receiving reimbursement for travel expenses from Sanofi-Aventis; Dr. Pinter, receiving consulting and lecture fees from Roche; Dr. Valero, receiving honoraria and reimbursement for travel expenses from Roche; Dr. Sauter, receiving honoraria and reimbursement for travel expenses from Roche; Dr. von Minckwitz, receiving fees for expert testimony, lecture fees, and reimbursement for travel expenses from Roche and Sanofi-Aventis; Dr. Buyse, being an employee of and having an equity interest in the International Drug Development Institute; Dr. Bendahmane and Dr. Tabah-Fisch, being employees of Sanofi-Aventis; Dr. TabahFisch, receiving stock options from Sanofi-Aventis; and Dr. Crown, receiving honoraria and reimbursement for travel expenses from Roche and Sanofi-Aventis and that his institution has also received grant support from Roche on his behalf. No other potential conflict of interest relevant to this article was reported.

We thank the staff of the Breast Cancer International Research Group for their assistance with all aspects of the conduct and reporting of this study; all participating patients who gave their consent to be included in the study; Dr. Sandra Swain, chair of the data and safety monitoring committee, as well as all committee members; and Dr. Jonathan Plehn, chair of the cardiac safety monitoring committee, as well as all committee members. 


\section{References}

1. Yarden Y, Sliwkowski MX. Untangling the ErbB signalling network. Nat Rev Mol Cell Biol. 2001; 2:127-37. [PubMed: 11252954]

2. Slamon DJ, Clark GM, Wong SG, Levin WJ, Ullrich A, McGuire WL. Human breast cancer: correlation of relapse and survival with amplification of the HER-2/neu oncogene. Science. 1987; 235:177-82. [PubMed: 3798106]

3. Slamon DJ, Godolphin W, Jones LA, et al. Studies of the HER-2/neu proto-onco-gene in human breast and ovarian cancer. Science. 1989; 244:707-12. [PubMed: 2470152]

4. Arboleda MJ, Lyons JF, Kabbinavar FF, et al. Overexpression of AKT2/protein kinase Bbeta leads to up-regulation of beta1 integrins, increased invasion, and metastasis of human breast and ovarian cancer cells. Cancer Res. 2003; 63:196-206. [PubMed: 12517798]

5. Benz CC, Scott GK, Sarup JC, et al. Estrogen-dependent, tamoxifen-resistant tumorigenic growth of MCF-7 cells transfected with HER2/neu. Breast Cancer Res Treat. 1992; 24:85-95. [PubMed: 8095168]

6. De Luca A, Carotenuto A, Rachiglio A, et al. The role of the EGFR signaling in tumor microenvironment. J Cell Physiol. 2008; 214:559-67. [PubMed: 17894407]

7. Feigin ME, Muthuswamy SK. ErbB receptors and cell polarity: new pathways and paradigms for understanding cell migration and invasion. Exp Cell Res. 2009; 315:707-16. [PubMed: 19022245]

8. Izumi Y, Xu L, di Tomaso E, Fukumura D, Jain RK. Tumour biology: herceptin acts as an antiangiogenic cocktail. Nature. 2002; 416:279-80. [PubMed: 11907566]

9. Marcotte R, Muller WJ. Signal transduction in transgenic mouse models of human breast cancer implications for human breast cancer. J Mammary Gland Biol Neoplasia. 2008; 13:323-35. [PubMed: 18651209]

10. Pietras RJ, Arboleda J, Reese DM, et al. HER-2 tyrosine kinase pathway targets estrogen receptor and promotes hormone-independent growth in human breast cancer cells. Oncogene. 1995; 10:2435-46. [PubMed: 7784095]

11. She QB, Chandarlapaty S, Ye Q, et al. Breast tumor cells with PI3K mutation or HER2 amplification are selectively addicted to Akt signaling. PLoS ONE. 2008; 3(8):e3065. [PubMed: 18725974]

12. Hudziak RM, Lewis GD, Winget M, Fendly BM, Shepard HM, Ullrich A. p185HER2 monoclonal antibody has anti-proliferative effects in vitro and sensitizes human breast tumor cells to tumor necrosis factor. Mol Cell Biol. 1989; 9:1165-72. [PubMed: 2566907]

13. Hancock MC, Langton BC, Chan T, et al. A monoclonal antibody against the c-erbB-2 protein enhances the cytotoxicity of cis-diamminedichloroplatinum against human breast and ovarian tumor cell lines. Cancer Res. 1991; 51:4575-80. [PubMed: 1678683]

14. Pegram MD, Slamon DJ. Combination therapy with trastuzumab (Herceptin) and cisplatin for chemoresistant metastatic breast cancer: evidence for receptor-enhanced chemosensitivity. Semin Oncol. 1999; 26(Suppl 12):89-95. [PubMed: 10482199]

15. Pietras RJ, Fendly BM, Chazin VR, Pegram MD, Howell SB, Slamon DJ. Antibody to HER-2/neu receptor blocks DNA repair after cisplatin in human breast and ovarian cancer cells. Oncogene. 1994; 9:1829-38. [PubMed: 7911565]

16. Slamon, DJ. Final Study Report (FSR) for H0407g: A Phase 1 safety and tolerance study of intravenous anti-p185 HER2 humanized monoclonal antibody (rhu MAb HER2) in patients with HER2 overexpressing tumors. U.S. Food and Drug Administration; 1996.

17. Carter P, Presta L, Gorman CM, et al. Humanization of an anti-p185HER2 antibody for human cancer therapy. Proc Natl Acad Sci U S A. 1992; 89:4285-9. [PubMed: 1350088]

18. Pegram MD, Pienkowski T, Northfelt DW, et al. Results of two open-label, multicenter phase II studies of docetaxel, platinum salts, and trastuzumab in HER2-positive advanced breast cancer. J Natl Cancer Inst. 2004; 96:759-69. [PubMed: 15150304]

19. Robert N, Leyland-Jones B, Asmar L, et al. Randomized phase III study of trastuzumab, paclitaxel, and carboplatin compared with trastuzumab and paclitaxel in women with HER-2-overexpressing metastatic breast cancer. J Clin Oncol. 2006; 24:2786-92. [PubMed: 16782917] 
20. Tripathy D, Slamon DJ, Cobleigh M, et al. Safety of treatment of metastatic breast cancer with trastuzumab beyond disease progression. J Clin Oncol. 2004; 22:1063-70. [PubMed: 15020607]

21. Slamon DJ, Leyland-Jones B, Shak S, et al. Use of chemotherapy plus a monoclonal antibody against HER2 for metastatic breast cancer that overexpresses HER2. N Engl J Med. 2001; 344:783-92. [PubMed: 11248153]

22. Piccart-Gebhart MJ, Procter M, Leyland-Jones B, et al. Trastuzumab after adjuvant chemotherapy in HER2-positive breast cancer. N Engl J Med. 2005; 353:1659-72. [PubMed: 16236737]

23. Romond EH, Perez EA, Bryant J, et al. Trastuzumab plus adjuvant chemotherapy for operable HER2-positive breast cancer. N Engl J Med. 2005; 353:1673-84. [PubMed: 16236738]

24. Joensuu H, Kellokumpu-Lehtinen P-L, Bono P, et al. Adjuvant docetaxel or vinorelbine with or without trastuzumab for breast cancer. N Engl J Med. 2006; 354:809-20. [PubMed: 16495393]

25. Smith I, Procter M, Gelber RD, et al. 2-Year follow-up of trastuzumab after adjuvant chemotherapy in HER2-positive breast cancer: a randomised controlled trial. Lancet. 2007; 369:29-36. [PubMed: 17208639]

26. Tan-Chiu E, Yothers G, Romond E, et al. Assessment of cardiac dysfunction in a randomized trial comparing doxorubicin and cyclophosphamide followed by paclitaxel, with or without trastuzumab as adjuvant therapy in node-positive, human epidermal growth factor receptor 2overexpressing breast cancer: NSABP B-31. J Clin Oncol. 2005; 23:7811-9. [PubMed: 16258083]

27. Pegram M, Hsu S, Lewis G, et al. Inhibitory effects of combinations of HER-2/neu antibody and chemotherapeutic agents used for treatment of human breast cancers. Oncogene. 1999; 18:224151. [PubMed: 10327070]

28. Pegram MD, Konecny GE, O'Callaghan C, Beryt M, Pietras R, Slamon DJ. Rational combinations of trastuzumab with chemotherapeutic drugs used in the treatment of breast cancer. J Natl Cancer Inst. 2004; 96:739-49. [PubMed: 15150302]

29. Pegram M, Forbes J, Pienkowski T, et al. BCIRG 007: first overall survival analysis of randomized phase III trial of trastuzumab plus docetaxel with or without carboplatin as first line therapy in HER2 amplified metastatic breast cancer (MBC). J Clin Oncol. 2007; 25(Suppl II):964s. abstract.

30. Press MF, Sauter G, Buyse M, et al. Alteration of topoisomerase II-alpha gene in human breast cancer: association with responsiveness to anthracycline-based chemotherapy. J Clin Oncol. 2011; 29:859-67. [PubMed: 21189395]

31. O'Malley FP, Chia S, Tu D, et al. Topoisomerase II alpha and responsiveness of breast cancer to adjuvant chemotherapy. J Natl Cancer Inst. 2009; 101:644-50. [PubMed: 19401546]

32. Slamon DJ, Press MF. Alterations in the TOP2A and HER2 genes: association with adjuvant anthracycline sensitivity in human breast cancers. J Natl Cancer Inst. 2009; 101:615-8. [PubMed: 19401550]

33. van de Vijver MJ, He YD, van 't Veer LJ, et al. A gene-expression signature as a predictor of survival in breast cancer. N Engl J Med. 2002; 347:1999-2009. [PubMed: 12490681]

34. Early Breast Cancer Trialists' Collaborative Group. Polychemotherapy for early breast cancer: an overview of the randomised trials. Lancet. 1998; 352:930-42. [PubMed: 9752815]

35. Dressler LG, Berry DA, Broadwater G, et al. Comparison of HER2 status by fluorescence in situ hybridization and immunohistochemistry to predict benefit from dose escalation of adjuvant doxorubicin-based therapy in node-positive breast cancer patients. J Clin Oncol. 2005; 23:428797. [PubMed: 15994142]

36. Muss HB, Thor AD, Berry DA, et al. c-erbB-2 expression and response to adjuvant therapy in women with node-positive early breast cancer. N Engl J Med. 1994; 330:1260-6. Erratum, N Engl J Med 1994; 331:211. [PubMed: 7908410]

37. Paik S, Bryant J, Park C, et al. erbB-2 and response to doxorubicin in patients with axillary lymph node-positive, hormone receptor-negative breast cancer. J Natl Cancer Inst. 1998; 90:1361-70. [PubMed: 9747867]

38. Paik S, Bryant J, Tan-Chiu E, et al. HER2 and choice of adjuvant chemotherapy for invasive breast cancer: National Surgical Adjuvant Breast and Bowel Project Protocol B-15. J Natl Cancer Inst. 2000; 92:1991-8. [PubMed: 11121461]

39. Pritchard KI, Shepherd LE, O'Malley FP, et al. HER2 and responsiveness of breast cancer to adjuvant chemotherapy. N Engl J Med. 2006; 354:2103-11. [PubMed: 16707747] 
40. Gennari A, Sormani MP, Pronzato P, et al. HER2 status and efficacy of adjuvant anthracyclines in early breast cancer: a pooled analysis of randomized trials. J Natl Cancer Inst. 2008; 100:14-20. [PubMed: 18159072]

41. Doyle JJ, Neugut AI, Jacobson JS, Grann VR, Hershman DL. Chemotherapy and cardiotoxicity in older breast cancer patients: a population-based study. J Clin Oncol. 2005; 23:8597-605. [PubMed: 16314622]

42. Pinder MC, Duan Z, Goodwin JS, Hortobagyi GN, Giordano SH. Congestive heart failure in older women treated with adjuvant anthracycline chemotherapy for breast cancer. J Clin Oncol. 2007; 25:3808-15. [PubMed: 17664460]

43. Hershman D, Neugut AI, Jacobson JS, et al. Acute myeloid leukemia or myelodysplastic syndrome following use of granulocyte colony-stimulating factors during breast cancer adjuvant chemotherapy. J Natl Cancer Inst. 2007; 99:196-205. [PubMed: 17284714] 


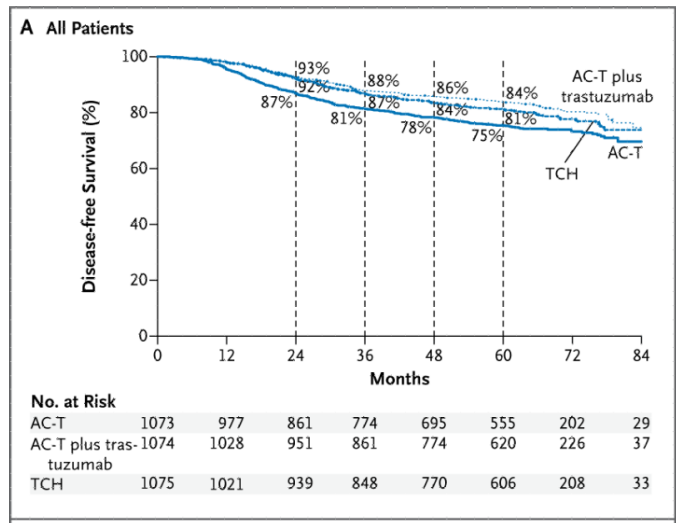

B Without TOP2A
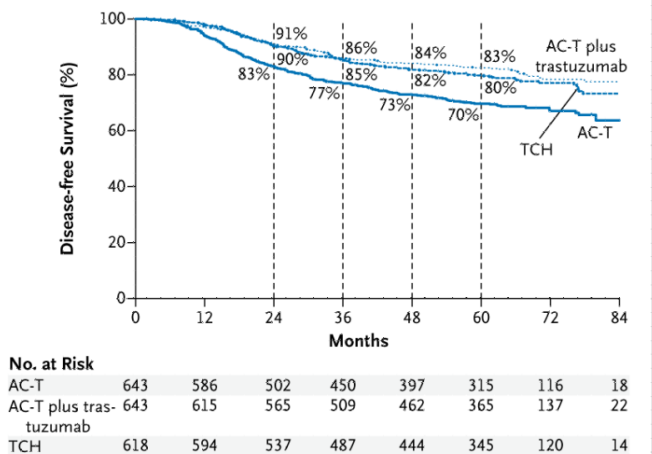

$\begin{array}{lllllllll}\begin{array}{ccccc}\text { tuzumab } \\ \text { TCH }\end{array} & 618 & 594 & 537 & 487 & 444 & 345 & 120 & 14\end{array}$

C With TOP2A
$95 \% \quad$ AC-T plus

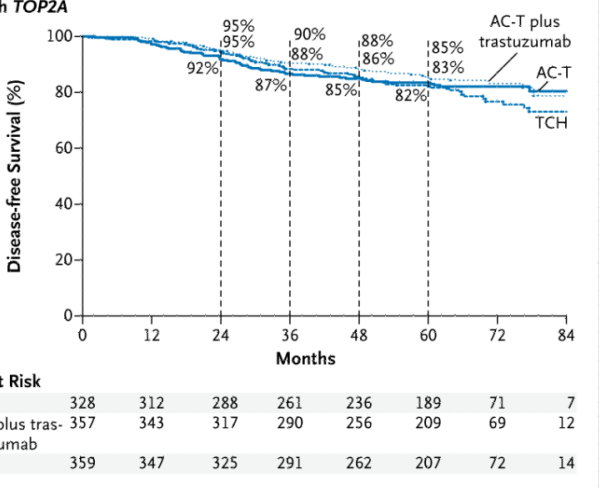

Figure 1. Disease-free Survival among All Patients and According to TOP2A Status

Shown are the results of Kaplan-Meyer analyses of relative rates of disease-free survival among all study patients (Panel A), those without coamplification of the gene encoding topoisomerase II alpha (TOP2A) (Panel B), and those with coamplification of TOP2A (Panel C). AC-T denotes doxorubicin and cyclophosphamide followed by docetaxel, and TCH docetaxel, carboplatin, and trastuzumab. 


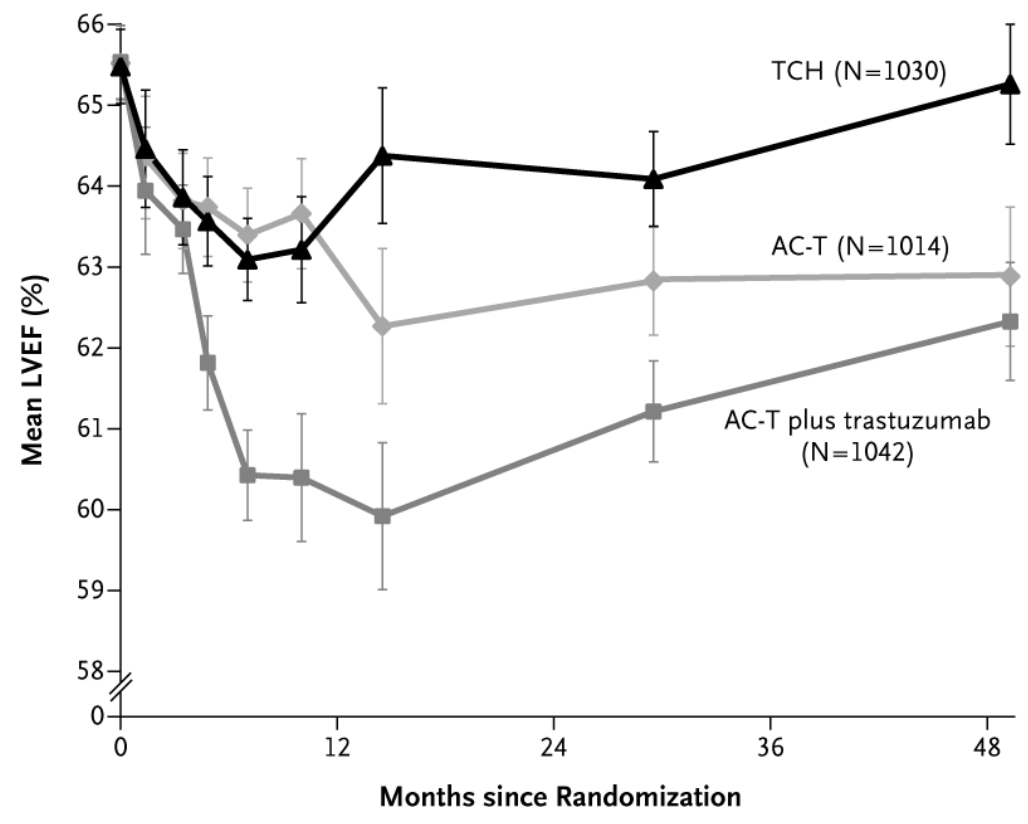

Figure 2. Left Ventricular Ejection Fraction (LVEF) at 48 Months

Shown are the values for the mean left ventricular ejection fraction for 3086 of 3222 patients $(96 \%)$ in the three study groups. At the time of this analysis, sufficient numbers of LVEF determinations were not yet available beyond 48 months. AC-T denotes doxorubicin and cyclophosphamide followed by docetaxel, and TCH docetaxel, carboplatin, and trastuzumab. 
Table 1

Baseline Characteristics of the Patients.*

\begin{tabular}{|lccc|}
\hline Characteristic & AC-T (N= 1073) & $\begin{array}{c}\text { AC-T plus Trastuzumab (N = 1074) } \\
\text { number of patients (percent) }\end{array}$ & TCH (N = 1075) \\
Age $<50 \mathrm{yr}$ & $562(52)$ & $559(52)$ & $577(54)$ \\
Karnofsky performance score of $100^{\dagger}$ & $856(80)$ & $853(79)$ & $862(80)$ \\
Mastectomy & $638(59)$ & $675(63)$ & $642(60)$ \\
Radiotherapy & $718(67)$ & $723(67)$ & $729(68)$ \\
Hormonal therapy & $531(49)$ & $542(50)$ & $541(50)$ \\
No. of positive nodes & & & $307(29)$ \\
0 & $309(29)$ & $306(28)$ & $415(39)$ \\
1 to 3 & $413(38)$ & $410(38)$ & $249(23)$ \\
4 to 10 & $235(22)$ & $259(24)$ & $104(10)$ \\
$>10$ & $116(11)$ & $99(9)$ & $431(40)$ \\
Tumor size & & & $574(53)$ \\
$\leq 2 \mathrm{~cm}$ & & $413(38)$ & $68(6)$ \\
$>2$ to 5 cm & $439(41)$ & $591(55)$ & $579(54)$ \\
$>5 \mathrm{~cm}$ & $566(53)$ & $70(7)$ & $578(54)$ \\
Positive for estrogen receptor, progesterone receptor, or both & $576(54)$ & & 5 \\
\hline
\end{tabular}

There were no significant differences among the three groups. AC-T denotes doxorubicin and cyclophosphamide followed by docetaxel, and TCH docetaxel, carboplatin, and trastuzumab. - $\mathbf{j}$ - A Karnofsky score of 100 indicates no measurable loss of performance status.

The tumor size was unavailable for one patient in the AC-T group and two patients in the TCH group. 
Table 2

Therapeutic Index for Critical Clinical Events.*

\begin{tabular}{|lrrr|}
\hline Clinical Event & AC-T & AC-T plus Trastuzumab & TCH \\
& \multicolumn{3}{c}{ number of events } \\
Total events & 201 & 146 & 149 \\
Distant breast-cancer recurrence & 188 & 124 & 144 \\
Grade 3 or 4 congestive heart failure & 7 & 21 & 4 \\
Acute leukemia & 6 & 1 & $1^{\dagger}$ \\
\hline
\end{tabular}

This therapeutic index is a compilation of the numbers of distant breast-cancer recurrences, cases of congestive heart failure, and cases of acute leukemia. AC-T denotes doxorubicin and cyclophosphamide followed by docetaxel, and TCH docetaxel, carboplatin, and trastuzumab.

${ }^{\dagger}$ This case of acute leukemia developed after the patient received an anthracy-cline as part of a combination chemotherapy regimen for a diffuse large B-cell lymphoma that occurred after she received treatment with $\mathrm{TCH}$ for breast cancer. 
Table 3

Adverse Events.*

\begin{tabular}{|c|c|c|c|c|}
\hline Event & AC-T $(N=1050)$ & $\begin{array}{r}\text { AC-T plus Trastuzumab }(\mathbf{N}=\mathbf{1 0 6 8}) \\
\text { number of patients (percent) }\end{array}$ & TCH $(N=1056)$ & P Value ${ }^{t}$ \\
\hline \multicolumn{5}{|c|}{ Grade 3 or 4 nonhematologic events } \\
\hline Arthralgia & $34(3.2)$ & $35(3.3)$ & $15(1.4)$ & 0.006 \\
\hline Myalgia & $55(5.2)$ & $56(5.2)$ & $19(1.8)$ & $<0.001$ \\
\hline Fatigue & $73(7.0)$ & $77(7.2)$ & $76(7.2)$ & 1.0 \\
\hline Hand-foot syndrome & $20(1.9)$ & $20(1.9)$ & 0 & $<0.001$ \\
\hline Stomatitis & $37(3.5)$ & $31(2.9)$ & $15(1.4)$ & 0.02 \\
\hline Diarrhea & $32(3.0)$ & $60(5.6)$ & $57(5.4)$ & 0.85 \\
\hline Nausea & $62(5.9)$ & $61(5.7)$ & $51(4.8)$ & 0.38 \\
\hline Vomiting & $65(6.2)$ & $72(6.7)$ & $37(3.5)$ & $<0.001$ \\
\hline Irregular menses & $284(27.0)$ & $260(24.3)$ & $280(26.5)$ & 0.25 \\
\hline \multicolumn{5}{|l|}{ Specific nonhematologic events } \\
\hline \multicolumn{5}{|l|}{ Neuropathy, any grade } \\
\hline Sensory & $510(48.6)$ & $531(49.7)$ & $380(36.0)$ & $<0.001$ \\
\hline Motor & $55(5.2)$ & $67(6.3)$ & $45(4.3)$ & 0.04 \\
\hline Nail changes, any grade & $518(49.3)$ & $466(43.6)$ & $303(28.7)$ & $<0.001$ \\
\hline Myalgia, any grade & $555(52.9)$ & $593(55.5)$ & $411(38.9)$ & $<0.001$ \\
\hline Renal failure, any grade & 0 & 0 & $1(0.1)$ & 0.50 \\
\hline Grade 3 or 4 creatinine elevation & $6(0.6)$ & $3(0.3)$ & $1(0.1)$ & 0.62 \\
\hline \multicolumn{5}{|l|}{ Grade 3 or 4 hematologic events } \\
\hline Neutropenia & $665(63.3)$ & $764(71.5)$ & $696(65.9)$ & 0.01 \\
\hline Leukopenia & $544(51.8)$ & $644(60.3)$ & $509(48.2)$ & $<0.001$ \\
\hline Febrile neutropenia & $98(9.3)$ & $116(10.9)$ & $101(9.6)$ & 0.35 \\
\hline Neutropenic infection & $117(11.1)$ & $127(11.9)$ & $118(11.2)$ & 0.63 \\
\hline Anemia & $25(2.4)$ & $33(3.1)$ & $61(5.8)$ & $<0.001$ \\
\hline Thrombocytopenia & $17(1.6)$ & $22(2.1)$ & $64(6.1)$ & $<0.001$ \\
\hline Leukemia & $6(0.6)$ & $1(0.1)$ & $1(0.1)$ & 1.0 \\
\hline
\end{tabular}

* AC-T denotes doxorubicin and cyclophosphamide followed by docetaxel, and TCH docetaxel, carboplatin, and trastuzumab.

${ }^{\dagger} \mathrm{P}$ values are for the comparison between the group receiving AC-T plus trastuzumab and the group receiving TCH.

Frade 3 or 4 creatinine elevation was defined according to the National Cancer Institute Common Toxicity Criteria, version 2.0. 
Table 4

Cardiac Risk Factors and Events. ${ }^{*}$

\begin{tabular}{|c|c|c|c|}
\hline Variable & AC-T $(N=1073)$ & $\begin{array}{c}\text { AC-T plus Trastuzumab }(\mathbf{N}=\mathbf{1 0 7 4}) \\
\text { number of patients }(\text { percent })\end{array}$ & TCH $(N=1075)$ \\
\hline \multicolumn{4}{|l|}{ Risk factors } \\
\hline Diabetes & $38(3.5)$ & $36(3.4)$ & $28(2.6)$ \\
\hline Hypertension & $178(16.6)$ & $178(16.6)$ & $190(17.7)$ \\
\hline Obesity $^{\dagger}$ & $214(19.9)$ & $242(22.5)$ & $234(21.8)$ \\
\hline Hypercholesterolemia & $54(5.0)$ & $47(4.4)$ & $43(4.0)$ \\
\hline Left-side radiotherapy & $378(35.2)$ & $349(32.5)$ & $364(33.9)$ \\
\hline \multicolumn{4}{|l|}{ Events } \\
\hline Cardiac-related death & 0 & 0 & 0 \\
\hline Congestive heart failure ${ }^{\ddagger}$ & $7(0.7)$ & $21(2.0)$ & $4(0.4)^{\S}$ \\
\hline$>10 \%$ relative reduction in left ventricular ejection fraction ${ }^{I /}$ & $114(11.2)^{/ /}$ & $194(18.6)$ & $97(9.4)^{* *}$ \\
\hline
\end{tabular}

* AC-T denotes doxorubicin and cyclophosphamide followed by docetaxel, and TCH docetaxel, carboplatin, and trastuzumab.

${ }^{\dagger}$ Obesity was defined as a body-mass index (the weight in kilograms divided by the square of the height in meters) of 30 or more.

This condition was defined as New York Heart Association grade 3 or 4 congestive heart failure.

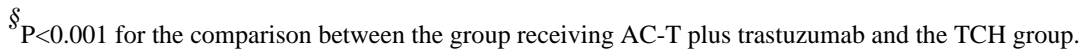

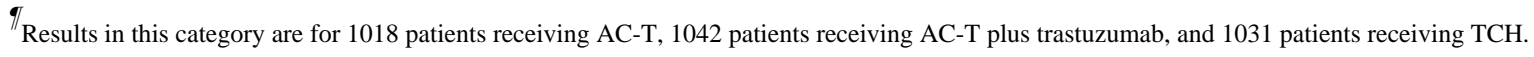

" $\mathrm{P}<0.001$ for the comparison between the group receiving AC-T plus trastuzumab and the AC-T group.

${ }_{\mathrm{P}}^{* *}<0.001$ for the comparison between the group receiving AC-T plus trastuzumab and the $\mathrm{TCH}$ group. 